\title{
Charge and longitudinal momentum distributions in transverse coordinate space
}

\author{
Chandan Mondal ${ }^{1}$, Narinder Kumar ${ }^{1,2}$, Harleen Dahiya ${ }^{2}$, Dipankar Chakrabarti $^{1}$ \\ ${ }^{1}$ Department of Physics, Indian Institute of Technology Kanpur, Kanpur-208016, India. \\ ${ }^{2}$ Department of Physics, Dr. B.R. Ambedkar National \\ Institute of Technology, Jalandhar-144011, India.
}

(Dated: October 4, 2018)

\begin{abstract}
We investigate the charge distributions for the $u$ and $d$ quarks in transverse coordinate space in a light-front quark-diquark model for the nucleons using the overlaps of the wave functions constructed from the soft-wall AdS/QCD prediction. We have also obtained the charge distributions for proton and neutron in transverse coordinate space and compared it with the distributions obtained in impact-parameter space. Further, we study the longitudinal momentum distributions using the wave functions in the transverse coordinate space. We have also shown the explicit fermionic and bosonic contributions for different struck $u$ and $d$ quarks.
\end{abstract}

PACS numbers: 12.38.Aw, 12.39.-x, 21.10.Ft 


\section{INTRODUCTION}

Hadronic structure and their properties, being nonperturbative in nature, are difficult to understand from the first principle of Quantum Chromodynamics (QCD). In the last decade, there have been numerous attempts to gain insight into the hadronic structure by studying the QCD inspired models [1 4] $]$. The quark-diquark model [5] is one of the most successful QCD inspired models to investigate various aspects of hardonic properties where the nucleon is considered to be a bound state of a single quark and a scalar or vector diquark state. Recently, a light-front scalar quark-diquark model for the nucleons inspired by soft-wall AdS/QCD has been proposed [6] and extensively used to investigate and reproduce many interesting properties of the nucleons [7-12]. The light-front wave functions (LFWFs) in this model are obtained by matching the electromagnetic form factors of the hadrons in the soft-wall model of AdS/QCD which has been successful in explaining various hadronic properties, for example, hadron mass spectrum, Parton Distribution Functions (PDFs), Generalized Parton Distributions (GPDs), meson and nucleon form factors, transverse densities, structure functions etc. [13 31$]$.

The AdS/CFT correspondence [32] between the string theories of gravity in the AdS space and conformal gauge field theories in the physical space-time provides a completely new set of tool for studying the dynamics of QCD. One can represent the strong interactions of quarks and gluons by a semi-classical gravity theory i.e., without quantum effects such as particle creation and annihilation in higher dimensions. Even though, a perfect string theory dual of QCD is not yet known, the AdS/CFT correspondence can still provide remarkable insight into various properties/features of QCD including color confinement, qualitative explanation for meson and baryon spectra and wave functions describing the hadron structure. QCD is conformal in the ultraviolet (UV) region, whereas, in the infrared (IR) region, the confining gauge theory with a mass gap is characterized by the scale $\Lambda_{Q C D}$ and a well defined spectrum of hadronic states. Light-front holography represents a remarkable connection between the AdS space and the light-front framework where the wave amplitude propagating in the AdS space is mapped into the light-front wave functions of hadrons in space-time in terms of a light-front transverse variable $\zeta$ (giving the separation of the quarks and gluonic constituents within the hadron) [33], 34].

The electromagnetic form factors, probed through elastic scattering, contain information 
about the internal structure of the nucleons. A Fourier transformation of these form factors provides information about the spatial distributions of charge. The well known examples include the charge and magnetization distributions inside the nucleons. One can obtain the Dirac (charge) and Pauli (magnetic) form factors from the overlap of the LFWFs by calculating the matrix element of electromagnetic current $J^{+}$in the light-front frame [35, 36]. In such a frame, the momentum transfer is the conjugate variable of the impact-parameter and via Fourier transform with respect to the momentum transfer in transverse direction, the form factors reflect the charge distributions of quarks in the impact-parameter space [9, 37-45]. The charge distributions in the transverse coordinate space obtained form the overlap of LFWFs in coordinate space have been studied [30, 46] and a comparison of charge distributions in transverse impact-parameter space in different AdS/QCD models has been reported in Refs. [8, 25].

The gravitational form factors related to the energy momentum tensor $\left(T^{\mu \nu}\right)$ also play an important role in the understanding of hadronic physics. They have been studied in both the light-cone and the AdS/QCD framework and can also be obtained from the second moments of GPDs [10, 26, 47 52]. The gravitational form factors $A\left(Q^{2}\right)$ and $B\left(Q^{2}\right)$ can be obtained from helicity non-flip and helicity flip matrix elements of the $T^{++}$tensor current similar to the Dirac $\left(F_{1}\left(Q^{2}\right)\right)$ and Pauli $\left(F_{2}\left(Q^{2}\right)\right)$ form factors respectively. The transverse spin sum rule and gravitational form factors have been studied [10]. The Fourier transform of the gravitational form factor $A\left(Q^{2}\right)$ in the impact-parameter space has an interesting interpretation [28, 40] as it gives the longitudinal momentum density $\left(p^{+}\right.$density) in the transverse impact-parameter space. A comparative study of longitudinal momentum densities in transverse impact-parameter space in two different soft-wall AdS/QCD models has been carried out [26].

The authors in Ref. [46] had introduced the charge distribution in transverse coordinate space and explicitly calculated the distribution considering a light-front quark-diquark model where they had used the modified wavefuntions of QED Yukawa model. Similar to charge distribution in transverse coordinate space, the distribution of longitudinal momentum $\left(p^{+}\right.$distribution $)$in the transverse coordinate space can also be investigated. It would be interesting to evaluate these distributions in the AdS/QCD framework which is one of the most successful nonperturbative tools to study the hadronic properties. In the present work, we study the charge as well as the longitudinal momentum distributions in 
the transverse coordinate space in a light-front quark-diquark model inspired by soft-wall AdS/QCD and compare the consequences with the results obtained in [46]. We take the phenomenological light-front quark-diquark model proposed by Gutsche et. al [6] with the parameters given in Ref. [7]. In this model, the LFWFs for the proton are constructed from the two particle wave functions obtained in soft-wall AdS/QCD [20]. The parameters in this model are fixed by fitting to the electromagnetic form factors of the nucleons. To obtain the charge distribution in coordinate space, we have taken the Fourier transform of LFWFs in momentum space. These wave functions have been used to obtain the charge distribution in coordinate space. Using the charge and isospin symmetry, we have also calculated the charge distributions for proton and neutron. We have also presented the result for longitudinal momentum distribution for $u$ and $d$ quarks. We have considered the different struck quarks to obtain longitudinal momentum distributions. Explicit results of the fermionic and bosonic contributions for longitudinal momentum distributions in coordinate space have been presented. It is important to mention here that even though both $r$ and $\left|b_{\perp}\right|$ are conjugate to momentum $k$ and momentum transferred $\Delta_{\perp}$ respectively, still the density in impact parameter space cannot be interpreted as coordinate space density. The quark-scalar diquark model has been shown to reproduce many interesting nucleon properties [6 12], but since only scalar diquark is considered in this model, the model might not reproduce correctly the nucleon observables involving orbital angular momentum and spin of the quarks and diquarks.

The paper is organized as follows. In Section II, a brief introduction about the nucleons LFWFs of a quark-diquark model in AdS/QCD is given. We discuss the charge distributions in transverse coordinate space for the $u$ and $d$ quarks as well as the nucleons in Section III. In Section IV, we present the relation between the charge distributions in transverse coordinate space and the impact-parameter space. The results of longitudinal momentum distributions in transverse coordinate space for proton and the quarks and diquarks contributions to the distributions for different struck quarks $u$ and $d$ are presented in Section $\nabla$. Finally, we summarize our work in Section VI. 


\section{LIGHT-FRONT QUARK-DIQUARK MODEL CONSTRUCTED BY ADS/QCD}

Here we consider a light-front quark-diquark model for the nucleons [6] where the LFWFs are modeled from the soft-wall AdS/QCD solution. In this model, one can contemplate the three valence quarks of the nucleons as an effectively composite system composed of a fermion (quark) and a composite state of diquark (boson) based on one loop quantum fluctuations. The Dirac and Pauli form factors for quarks in this model can be evaluated in terms of overlap of the LFWFs [35, 36] as

$$
\begin{aligned}
F_{1}^{q}\left(Q^{2}\right) & =\int_{0}^{1} d x \int \frac{d^{2} \mathbf{k}_{\perp}}{16 \pi^{3}}\left[\psi_{+q}^{+*}\left(x, \mathbf{k}_{\perp}^{\prime}\right) \psi_{+q}^{+}\left(x, \mathbf{k}_{\perp}\right)+\psi_{-q}^{+*}\left(x, \mathbf{k}_{\perp}^{\prime}\right) \psi_{-q}^{+}\left(x, \mathbf{k}_{\perp}\right)\right] \\
F_{2}^{q}\left(Q^{2}\right) & =-\frac{2 M_{n}}{q^{1}-i q^{2}} \int_{0}^{1} d x \int \frac{d^{2} \mathbf{k}_{\perp}}{16 \pi^{3}}\left[\psi_{+q}^{+*}\left(x, \mathbf{k}_{\perp}^{\prime}\right) \psi_{+q}^{-}\left(x, \mathbf{k}_{\perp}\right)\right. \\
& \left.+\psi_{-q}^{+*}\left(x, \mathbf{k}_{\perp}^{\prime}\right) \psi_{-q}^{-}\left(x, \mathbf{k}_{\perp}\right)\right]
\end{aligned}
$$

with $\mathbf{k}_{\perp}^{\prime}=\mathbf{k}_{\perp}+(1-x) \mathbf{q}_{\perp}$ for the struck quark. Here $x$ is the light-cone momentum fraction and $\psi_{\lambda_{q} q}^{\lambda_{N}}\left(x, \mathbf{k}_{\perp}\right)$ are the LFWFs with nucleon helicities $\lambda_{N}= \pm$ and for the struck quark $\lambda_{q}= \pm$, where plus and minus correspond to $+\frac{1}{2}$ and $-\frac{1}{2}$ respectively. In the frame $q=\left(q^{+}, q^{-}, \mathbf{q}_{\perp}\right)=\left(0,0, \mathbf{q}_{\perp}\right)$, we have $Q^{2}=-q^{2}=\mathbf{q}_{\perp}^{2}$. The LFWFs defined at an initial scale $\mu_{0}=313 \mathrm{MeV}$ [6] are given as

$$
\begin{aligned}
\psi_{+q}^{+}\left(x, \mathbf{k}_{\perp}\right) & =\varphi_{q}^{(1)}\left(x, \mathbf{k}_{\perp}\right) \\
\psi_{-q}^{+}\left(x, \mathbf{k}_{\perp}\right) & =-\frac{k^{1}+i k^{2}}{x M_{n}} \varphi_{q}^{(2)}\left(x, \mathbf{k}_{\perp}\right), \\
\psi_{+q}^{-}\left(x, \mathbf{k}_{\perp}\right) & =\frac{k^{1}-i k^{2}}{x M_{n}} \varphi_{q}^{(2)}\left(x, \mathbf{k}_{\perp}\right) \\
\psi_{-q}^{-}\left(x, \mathbf{k}_{\perp}\right) & =\varphi_{q}^{(1)}\left(x, \mathbf{k}_{\perp}\right)
\end{aligned}
$$

where $\varphi_{q}^{(i=1,2)}\left(x, \mathbf{k}_{\perp}\right)$ are the modified wave functions constructed by soft-wall AdS/QCD, after introducing the parameters $a_{q}^{(i)}$ and $b_{q}^{(i)}$ for quark $q$ [6] and are defined as

$$
\varphi_{q}^{(i)}\left(x, \mathbf{k}_{\perp}\right)=N_{q}^{(i)} \frac{4 \pi}{\kappa} \sqrt{\frac{\log (1 / x)}{1-x}} x^{a_{q}^{(i)}}(1-x)^{b_{q}^{(i)}} \exp \left[-\frac{\mathbf{k}_{\perp}^{2}}{2 \kappa^{2}} \frac{\log (1 / x)}{(1-x)^{2}}\right] .
$$

For $a_{q}^{(i)}=b_{q}^{(i)}=0, \varphi_{q}^{(i)}\left(x, \mathbf{k}_{\perp}\right)$ reduces to the AdS/QCD solution [20]. In this work, we have taken the AdS/QCD scale parameter $\kappa=0.4 \mathrm{GeV}$, obtained by fitting the nucleon form factors in the AdS/QCD soft-wall model [23, 24]. All the parameters $a_{q}^{(i)}$ and $b_{q}^{(i)}$ with the constants $N_{q}^{(i)}$ are determined by fitting the electromagnetic properties of the nucleons: $F_{1}^{q}(0)=n_{q}$ and $F_{2}^{q}(0)=\kappa_{q}$ where the number of valence $u$ and $d$ quarks are $n_{u}=2$ and 
$n_{d}=1$ in proton and the anomalous magnetic moments for the $u$ and $d$ quarks are $\kappa_{u}=1.673$ and $\kappa_{d}=-2.033$ [7] . One can write the flavor decompositions of the Dirac and Pauli form factors of nucleons in a straightforward manner as

$$
F_{i}^{p(n)}=e_{u} F_{i}^{u(d)}+e_{d} F_{i}^{d(u)}, \quad(i=1,2)
$$

where $e_{u}$ and $e_{d}$ are the charges of $u$ and $d$ quarks in units of positron charge $(e)$. The nucleon form factors as well as the flavor form factors in this model have already been calculated in Ref. [8] and are found to agree very well with the experimental data.

\section{CHARGE DISTRIBUTIONS IN COORDINATE SPACE}

In order to evaluate the charge density in coordinate space, the LFWFs in the transverse coordinate space $\tilde{\psi}\left(x, r_{\perp}\right)$ can be obtained by taking the Fourier transform of the LFWFs in the momentum space $\psi\left(x, \mathbf{k}_{\perp}\right)$, as

$$
\tilde{\psi}\left(x, \mathbf{r}_{\perp}\right)=\int \frac{d^{2} \mathbf{k}_{\perp}}{(2 \pi)^{2}} e^{i \mathbf{k}_{\perp} \cdot \mathbf{r}_{\perp}} \psi\left(x, \mathbf{k}_{\perp}\right) .
$$

The charge distribution in transverse coordinate space for a particular flavor in the quarkdiquark model is defined as [46]

$$
P_{f}^{q}(r)=\int d x P_{f}^{q}(x, r)=\int d x\left[\tilde{\psi}_{+q}^{+*}\left(x, \mathbf{r}_{\perp}\right) \tilde{\psi}_{+q}^{+}\left(x, \mathbf{r}_{\perp}\right)+\tilde{\psi}_{-q}^{+*}\left(x, \mathbf{r}_{\perp}\right) \tilde{\psi}_{-q}^{+}\left(x, \mathbf{r}_{\perp}\right)\right]
$$

where $r=\left|\mathbf{r}_{\perp}\right|$. To obtain the charge distribution in transverse coordinate space in the light-front diquark model where the two-particle wave function is modeled from the softwall AdS/QCD, we first calculate the LFWFs in transverse coordinate space via Fourier transform of the wave functions given in Eq. (3)

$$
\begin{aligned}
\tilde{\psi}_{q+}^{+}\left(x, \mathbf{r}_{\perp}\right) & =\int \frac{d^{2} \mathbf{k}_{\perp}}{(2 \pi)^{2}} e^{i \mathbf{k}_{\perp} \cdot \mathbf{r}_{\perp}} \psi_{q+}^{+}\left(x, \mathbf{k}_{\perp}\right) \\
& =\frac{N_{q}^{1}}{2 \pi} \frac{4 \pi}{\kappa} \sqrt{\frac{\log (1 / x)}{1-x}} x^{a_{q}^{1}}(1-x)^{b_{q}^{1}} \frac{e^{-r^{2} / 4 g}}{2 g} \\
\tilde{\psi}_{q-}^{+}\left(x, \mathbf{r}_{\perp}\right) & =\int \frac{d^{2} \mathbf{k}_{\perp}}{(2 \pi)^{2}} e^{i \mathbf{k}_{\perp} \cdot \mathbf{r}_{\perp}} \psi_{-q}^{+}\left(x, \mathbf{k}_{\perp}\right) \\
& =-i \frac{N_{q}^{2}}{2 \pi} \frac{4 \pi}{\kappa} \sqrt{\frac{\log (1 / x)}{1-x}} \frac{x^{a_{q}^{2}}(1-x)^{b_{q}^{2}}}{M x} \frac{\left(r^{1}+i r^{2}\right) e^{\frac{-r^{2}}{4 g}}}{4 g^{2}} \\
\tilde{\psi}_{q+}^{-}\left(x, \mathbf{r}_{\perp}\right) & =\int \frac{d^{2} \mathbf{k}_{\perp}}{(2 \pi)^{2}} e^{i \mathbf{k}_{\perp} \cdot \mathbf{r}_{\perp}} \psi_{+q}^{-}\left(x, \mathbf{k}_{\perp}\right)
\end{aligned}
$$




$$
\begin{aligned}
& =i \frac{N_{q}^{2}}{2 \pi} \frac{4 \pi}{\kappa} \sqrt{\frac{\log (1 / x)}{1-x}} \frac{x^{a_{q}^{2}}(1-x)^{b_{q}^{2}}}{M x} \frac{\left(r^{1}-i r^{2}\right) e^{\frac{-r^{2}}{4 g}}}{4 g^{2}} \\
\tilde{\psi}_{q-}^{-}\left(x, \mathbf{r}_{\perp}\right) & =\int \frac{d^{2} \mathbf{k}_{\perp}}{(2 \pi)^{2}} e^{i \mathbf{k}_{\perp} \cdot \mathbf{r}_{\perp} \psi_{-q}^{-}\left(x, \mathbf{k}_{\perp}\right)} \\
& =\frac{N_{q}^{1}}{2 \pi} \frac{4 \pi}{\kappa} \sqrt{\frac{\log (1 / x)}{1-x}} \frac{x^{a_{q}^{2}}(1-x)^{b_{q}^{2}}}{M x} \frac{e^{\frac{-r^{2}}{4 g}}}{2 g}
\end{aligned}
$$

where $g=\frac{\log (1 / x)}{2 \kappa^{2}(1-x)^{2}}$. Substituting Eq.(8) in Eq.(17), we can evaluate the charge distributions for a particular flavor in transverse coordinate space and is given as

$$
P_{f}^{q}(r)=\int d x \frac{\log (1 / x)}{(1-x)}\left[\left(N_{q}^{1}\right)^{2} x^{2 a_{q}^{1}}(1-x)^{2 b_{q}^{1}}+\left(N_{q}^{2}\right)^{2} \frac{x^{2 a_{q}^{2}}(1-x)^{2 b_{q}^{2}}}{x^{2} M_{n}^{2}} \frac{r^{2}}{4 g^{2}}\right] \frac{1}{\kappa^{2} g^{2}} e^{\frac{-r^{2}}{4 g}} .
$$

As for the case of decomposition for Dirac and Pauli form factors in Eq.(5), using charge and isospin symmetry, we can write the charge distributions for the nucleons [25, 46] as follows

$$
\begin{aligned}
& P^{p}=e_{u} P_{f}^{u}+e_{d} P_{f}^{d}=\frac{4}{3} P^{u}-\frac{1}{3} P^{d}, \\
& P^{n}=e_{u} P_{f}^{d}+e_{d} P_{f}^{u}=\frac{2}{3} P^{d}-\frac{2}{3} P^{u},
\end{aligned}
$$

where $P_{f}^{q}(r)$ and $P^{q}$ are the charge distributions for a particular flavor present in the nucleon and the individual quark respectively. Thus, the individual quark distributions are related to the charge distribution of a particular flavor present in the proton is

$$
\begin{aligned}
& P^{u}=\frac{P_{f}^{u}}{2}, \\
& P^{d}=P_{f}^{d} .
\end{aligned}
$$

\section{CHARGE DENSITIES IN IMPACT-PARAMETER SPACE}

According to the standard interpretation [25, 37-40], the charge density in the impactparameter space can be identified with the two-dimensional Fourier transform of the Dirac form factor in the light-cone frame with $q^{+}=q^{0}+q^{3}=0$

$$
\rho_{c h}(b)=\int \frac{d^{2} \mathbf{q}_{\perp}}{(2 \pi)^{2}} F_{1}\left(q^{2}\right) e^{i \mathbf{q}_{\perp} \cdot \mathbf{b}_{\perp}},
$$

where the impact-parameter, $b=\left|\mathbf{b}_{\perp}\right|$. Using the Dirac form factor in term of overlaps of LFWFs defined in Eq.(11), we can write the charge density for a particular flavor in the nucleon as

$$
\begin{aligned}
\rho_{f c h}^{q}(b)= & \int \frac{d^{2} \mathbf{q}_{\perp}}{(2 \pi)^{2}} e^{i \mathbf{q}_{\perp} \cdot \mathbf{b}_{\perp}} \frac{1}{4 \pi} \int_{0}^{1} d x \int \frac{d^{2} \mathbf{k}_{\perp}}{(2 \pi)^{2}}\left[\psi_{+q}^{+*}\left(x, \mathbf{k}_{\perp}^{\prime}\right) \psi_{+q}^{+}\left(x, \mathbf{k}_{\perp}\right)\right. \\
& \left.+\psi_{-q}^{+*}\left(x, \mathbf{k}_{\perp}^{\prime}\right) \psi_{-q}^{+}\left(x, \mathbf{k}_{\perp}\right)\right] .
\end{aligned}
$$


Now, using the elementary theorem of convolutions and Fourier transform (see the appendix A), the above Eq.(13]) can be re-written as [46]

$$
\begin{aligned}
\rho_{f c h}^{q}(b)= & \frac{1}{4 \pi} \int_{0}^{1} d x \frac{1}{(1-x)^{2}}\left[\tilde{\psi}_{+q}^{+*}\left(x, \frac{\mathbf{b}_{\perp}}{x-1}\right) \tilde{\psi}_{+q}^{+}\left(x, \frac{\mathbf{b}_{\perp}}{x-1}\right)\right. \\
& \left.+\tilde{\psi}_{-q}^{+*}\left(x, \frac{\mathbf{b}_{\perp}}{x-1}\right) \tilde{\psi}_{-q}^{+}\left(x, \frac{\mathbf{b}_{\perp}}{x-1}\right)\right], \\
= & \frac{1}{4 \pi} \int_{0}^{1} d x \frac{1}{(1-x)^{2}} P_{f}^{q}\left(x, \frac{\mathbf{b}_{\perp}}{x-1}\right) .
\end{aligned}
$$

This relation shows that the coordinate space density is not the same as impact parameter space density. Though both $r$ and $\left|b_{\perp}\right|$ are conjugate to momenta, there is still a distinction between these two. $r$ is conjugate to the momentum $k$ whereas $b_{\perp}$ is conjugate to the momentum transferred $\Delta_{\perp}$. So, interpreting the density in impact parameter space as the coordinate space density is not correct. Similar calculations have been carried out in Ref. [46] where they have calculated the charge distribution in transverse coordinate space, however the factor $\frac{1}{4 \pi}$ is missing in the relation between charge distributions in impact-parameter space and the transverse coordinate space. Now, the charge densities for nucleons can be written in term of flavor densities [9, 25] as follows

$$
\begin{aligned}
& \rho_{c h}^{p}=e_{u} \rho_{f c h}^{u}+e_{d} \rho_{f c h}^{d}, \\
& \rho_{c h}^{n}=e_{u} \rho_{f c h}^{d}+e_{d} \rho_{f c h}^{u} .
\end{aligned}
$$

Due to the charge and isospin symmetry, the $u$ and $d$ quark densities in the proton are same as the $d$ and $u$ quark densities in the neutron [25, 37]. Under the charge and isospin symmetry, one can write

$$
\begin{aligned}
& \rho_{c h}^{u}(b)=\rho_{c h}^{p}+\frac{\rho_{c h}^{n}}{2}=\frac{\rho_{f c h}^{u}}{2}, \\
& \rho_{c h}^{d}(b)=\rho_{c h}^{p}+2 \rho_{c h}^{n}=\rho_{f c h}^{d},
\end{aligned}
$$

where $\rho_{c h}^{q}(b)$ is the charge density of each quark and $\rho_{f c h}^{q}$ is the charge density for a particular flavor.

In Fig,1, we show the charge distributions for $u$ and $d$ quarks in the transverse coordinate space. The charge distributions for $u$ and $d$ in impact-parameter space are also shown in the same plot. With $2 \sigma$ error in the model parameters, we estimate the errors in the model predictions. The bands in the plots show the error in our model. For $u$ quark the errors come out to be very small with $2 \sigma$ uncertainty in the model parameters. We observe that 
(a)

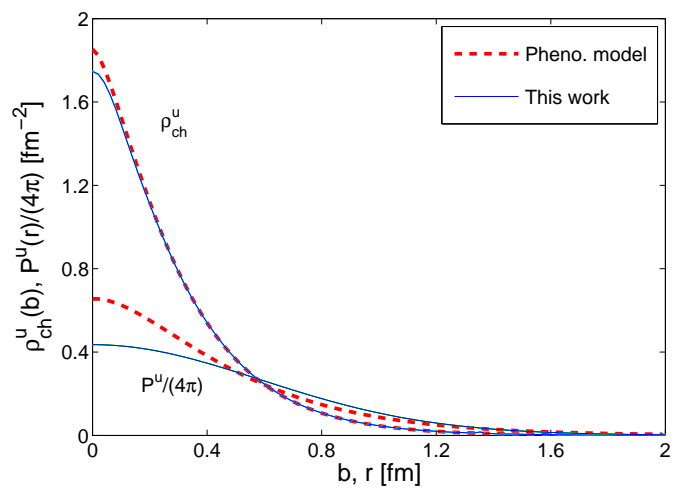

(b)

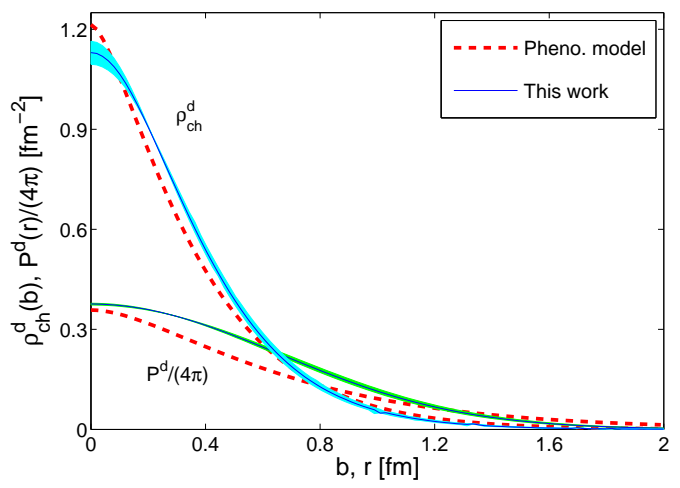

FIG. 1. (colour online) Charge distribution $P^{q}\left(\mathbf{r}_{\perp}\right)$ and $\rho_{c h}^{q}\left(\mathbf{b}_{\perp}\right)$ in the transverse coordinate and impact-parameter spaces respectively for the (a) $u$ quarks and (b) $d$ quarks, where $r=\left|\mathbf{r}_{\perp}\right|$ and $b=\left|\mathbf{b}_{\perp}\right|$. The blue solid lines represent the quark-diquark model in AdS/QCD (this work) and the red dashed lines represent the phenomenological model [46]. The bands in the plots represent the errors in the model predictions. For $u$ quark the bands are very small.

(a)

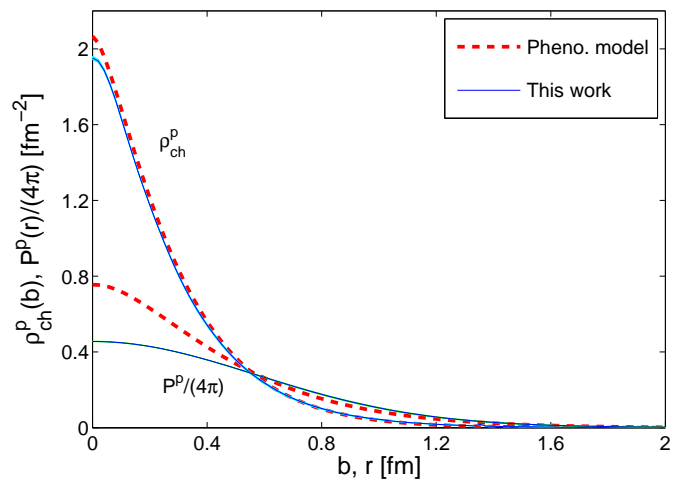

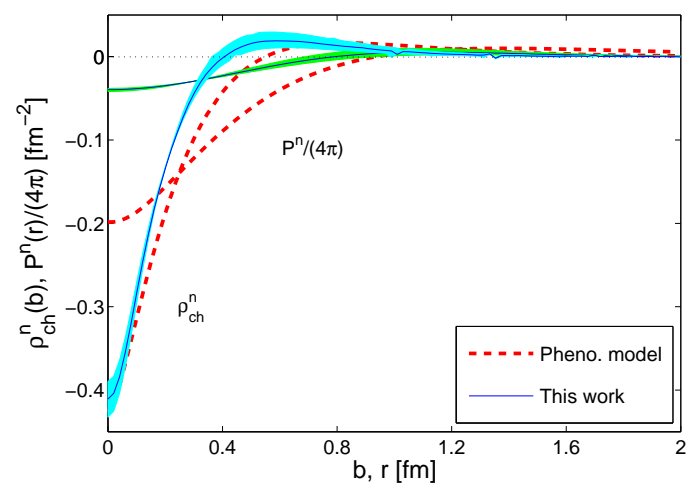

FIG. 2. (colour online) Charge distribution $P^{p, n}\left(\mathbf{r}_{\perp}\right)$ and $\rho_{c h}^{p, n}\left(\mathbf{b}_{\perp}\right)$ in the transverse coordinate and impact-parameter spaces respectively for the (a) proton and (b) neutron. The blue solid lines represent the quark-diquark model in AdS/QCD (this work) and the red dashed lines represent the phenomenological model [46]. The bands in the plots represent the errors in the model predictions.

the magnitude of charge distributions in impact-parameter is larger than the distributions in transverse coordinate space. The charge distributions for proton and neutron in both transverse coordinate and impact-parameter spaces are plotted in Fig 2 , Since these quantities are not directly measured in experiments, actual experimental data are not available. Thus, we compare the distributions for both quarks and nucleon with the results of another phenomenological model [46]. We observe that the quark-diquark model in AdS/QCD is 
(a)

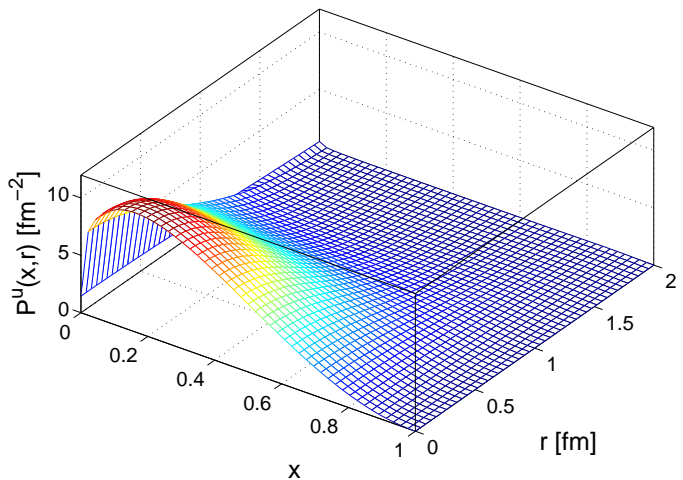

FIG. 3. (colour online) Charge distribution (a) $P^{u}\left(x, \mathbf{r}_{\perp}\right)$ and (b) $P^{d}\left(x, \mathbf{r}_{\perp}\right)$ in the transverse coordinate space as a function of $\mathbf{r}_{\perp}$ and $x$.

(a)

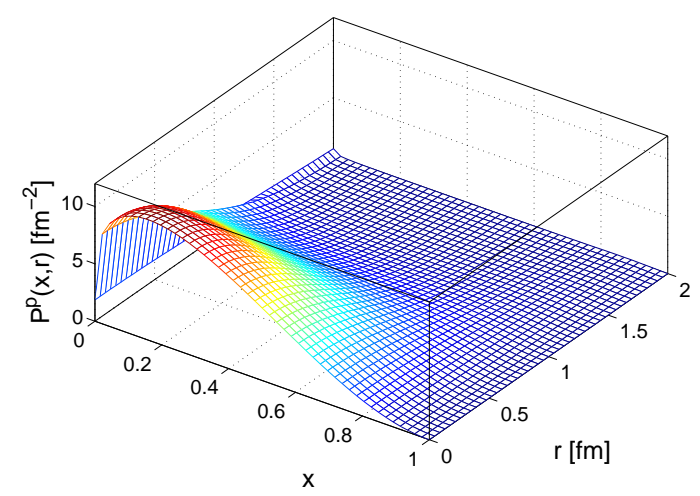

FIG. 4. (colour online) Charge distribution (a) $P^{p}\left(x, \mathbf{r}_{\perp}\right)$ and (b) $P^{n}\left(x, \mathbf{r}_{\perp}\right)$ in the transverse coordinate space as a function of $\mathbf{r}_{\perp}$ and $x$.

in good agreement with the phenomenological model for the charge distributions in impact parameter plane. Though, one can see from Fig.2(b), that the two models do not agree for the charge distributions for neutron and at low $\mathbf{r}_{\perp}$, the phenomenological model gives higher values of $P(r)$ (except $P^{d}$ ), the overall behaviors of the distributions in both the models are qualitatively the same. One can notice that the width of the distributions in coordinate space is larger than that in impact-parameter space. It can be understood from the relation between these two distributions shown in Eq.(14) where $\frac{\mathbf{b}_{\perp}}{x-1}$ appears in place of $\mathbf{r}_{\perp}$ in the argument of $P\left(x, \mathbf{r}_{\perp}\right)$. We also observe that the proton (neutron) charge density and distribution has a long range positively (negatively) charged component. The charge distribution for neutron is observed to be mostly negative though it also shows a slightly positive distribution whereas for proton it remains positive. This shows that charge distribution for 
neutron has a negatively charged core surrounded by a positively charged shell. For proton, we notice that, the density is peaked at low values of $b$ and has a long positive tail. Similar behavior of the charge distributions has been observed in other phenomenological models [30, 46].

In order to get more information about the distributions $P\left(x, \mathbf{r}_{\perp}\right)$, we plot the distributions as a function of $x$ and $\mathbf{r}_{\perp}$. In Fig 3 , we show the distributions $P\left(x, \mathbf{r}_{\perp}\right)$ for individual $u$ and $d$ quarks as a function of $x$ and $\mathbf{r}_{\perp}$. Similar plots for proton and neutron are shown in Fig 4. We observe that all the distributions are peaked at lower $x$ and with increasing the value of $x$, the magnitudes of the charge distributions decrease. However, the magnitude of the distributions for $u$ and $d$ quarks are more or less same.

\section{LONGITUDINAL MOMENTUM DISTRIBUTION: COORDINATE SPACE}

For a spin-half composite system, similar to the electromagnetic form factors, the gravitational form factors $A\left(Q^{2}\right)$ and $B\left(Q^{2}\right)$ can be obtained from the helicity conserving and helicity-flip matrix elements of the $T^{++}$tensor current. $A\left(Q^{2}\right)$ and $B\left(Q^{2}\right)$ are analogous to $F_{1}\left(Q^{2}\right)$ (Dirac) and $F_{2}\left(Q^{2}\right)$ (Pauli) form factors for the $J^{+}$vector current. The helicity conserved form factor $A\left(Q^{2}\right)$ allows one to measure the momentum fractions carried by each constituent of a hadron. The gravitational form factors in the light-front quark-diquark model can be obtained in terms of the overlap of the wave functions as [10, 36]

$$
\begin{aligned}
A^{q}\left(Q^{2}\right) & =\int_{0}^{1} d x \int \frac{d^{2} \mathbf{k}_{\perp}}{16 \pi^{3}} x\left[\psi_{+q}^{+*}\left(x, \mathbf{k}_{\perp}^{\prime}\right) \psi_{+q}^{+}\left(x, \mathbf{k}_{\perp}\right)+\psi_{-q}^{+*}\left(x, \mathbf{k}_{\perp}^{\prime}\right) \psi_{-q}^{+}\left(x, \mathbf{k}_{\perp}\right)\right] \\
B^{q}\left(Q^{2}\right) & =-\frac{2 M_{n}}{q^{1}-i q^{2}} \int_{0}^{1} d x \int \frac{d^{2} \mathbf{k}_{\perp}}{16 \pi^{3}} x\left[\psi_{+q}^{+*}\left(x, \mathbf{k}_{\perp}^{\prime}\right) \psi_{+q}^{-}\left(x, \mathbf{k}_{\perp}\right)\right. \\
& \left.+\psi_{-q}^{+*}\left(x, \mathbf{k}_{\perp}^{\prime}\right) \psi_{-q}^{-}\left(x, \mathbf{k}_{\perp}\right)\right] .
\end{aligned}
$$

The ++ component of the energy-momentum tensor $\left(T^{\mu \nu}\right)$ provides the longitudinal momentum

$$
P^{+}=\int d x^{-} d^{2} x^{\perp} T^{++}
$$

Using the LFWFs in transverse coordinate space we can now define the longitudinal momentum distribution in the transverse coordinate space for a struck flavor $q$ (fermionic)

$$
P_{L q(\text { flavor })}^{f}(r)=\int d x P_{L}^{q}(x, r)=\int d x x\left[\tilde{\psi}_{+q}^{+*}\left(x, \mathbf{r}_{\perp}\right) \tilde{\psi}_{+q}^{+}\left(x, \mathbf{r}_{\perp}\right)+\tilde{\psi}_{-q}^{+*}\left(x, \mathbf{r}_{\perp}\right) \tilde{\psi}_{-q}^{+}\left(x, \mathbf{r}_{\perp}\right)\right]
$$




$$
=\int d x x \frac{\log (1 / x)}{(1-x)}\left[\left(N_{q}^{1}\right)^{2} x^{2 a_{q}^{1}}(1-x)^{2 b_{q}^{1}}+\left(N_{q}^{2}\right)^{2} \frac{x^{2 a_{q}^{2}}(1-x)^{2 b_{q}^{2}}}{x^{2} M_{n}^{2}} \frac{r^{2}}{4 g^{2}}\right] \frac{1}{\kappa^{2} g^{2}} e^{\frac{-r^{2}}{4 g}},
$$

and for the diquark (bosonic)

$$
\begin{aligned}
P_{L q(f l a v o r)}^{b}(r) & =\int d x P_{L}^{b}(x, r)=\int d x(1-x)\left[\tilde{\psi}_{+q}^{+*}\left(x, \mathbf{r}_{\perp}\right) \tilde{\psi}_{+q}^{+}\left(x, \mathbf{r}_{\perp}\right)+\tilde{\psi}_{-q}^{+*}\left(x, \mathbf{r}_{\perp}\right) \tilde{\psi}_{-q}^{+}\left(x, \mathbf{r}_{\perp}\right)\right] \\
& =\int d x(1-x) \frac{\log (1 / x)}{(1-x)}\left[\left(N_{q}^{1}\right)^{2} x^{2 a_{q}^{1}}(1-x)^{2 b_{q}^{1}}+\left(N_{q}^{2}\right)^{2} \frac{x^{2 a_{q}^{2}}(1-x)^{2 b_{q}^{2}}}{x^{2} M_{n}^{2}} \frac{r^{2}}{4 g^{2}}\right] \frac{1}{\kappa^{2} g^{2}} e^{\frac{-r^{2}}{4 g}}
\end{aligned}
$$

Thus, the longitudinal momentum distributions for the proton in transverse coordinate space for the struck $u$ and $d$ quarks are given as

$$
\begin{aligned}
& P_{L u}^{p}(r)=\frac{1}{2}\left[P_{\text {Lu(flavor })}^{f}+P_{L u(\text { flavor })}^{b}\right]=P_{L u}^{f}+P_{L u}^{b}, \\
& P_{L d}^{p}(r)=\left[P_{L d(\text { flavor })}^{f}+P_{L d(\text { flavor })}^{b}\right]=P_{L d}^{f}+P_{L d}^{b} .
\end{aligned}
$$

For $P_{L u}^{p}$, the $\frac{1}{2}$ factor appears due to two possibilities of struck quark being a $u$ quark (two valence $u$ quark in proton).

As in the case of the electromagnetic form factors $F_{1}\left(q^{2}\right)$ and $F_{2}\left(q^{2}\right)$, the electromagnetic densities were defined from the Fourier transforms, one can in a similar manner interpret the two-dimensional Fourier transform of gravitational form factor $A\left(Q^{2}\right)$ as the longitudinal momentum densities in the impact-parameter plane [10, 26, 28, 40] as follows

$$
\rho_{L}(b)=\int \frac{d^{2} \mathbf{q}_{\perp}}{(2 \pi)^{2}} A\left(q^{2}\right) e^{i \mathbf{q}_{\perp} \cdot \mathbf{b}_{\perp}} .
$$

Using the gravitational form factor in Eq.(17) in terms of wave functions and the basic theorem of convolutions (see appendix A), we can relate the longitudinal momentum density $\rho_{L}(b)$ in the impact-parameter space with the density $P_{L}(r)$ in transverse coordinate space. For the quark (fermion), the longitudinal momentum density in the impact-parameter space is given as

$$
\rho_{L q}^{f}(b)=\frac{1}{4 \pi} \int_{0}^{1} d x \frac{1}{(1-x)^{2}} P_{L q}^{f}\left(x, \frac{b}{x-1}\right)=\frac{1}{4 \pi} \int_{0}^{1} d x \frac{x}{(1-x)^{2}} P_{f}^{q}\left(x, \frac{b}{x-1}\right) .
$$

For the diquark (boson), the longitudinal momentum density in the impact-parameter space is given $\mathrm{s}$

$$
\rho_{L q}^{b}(b)=\frac{1}{4 \pi} \int_{0}^{1} d x \frac{1}{x^{2}} P_{L q}^{b}\left(x, \frac{b}{x}\right)=\frac{1}{4 \pi} \int_{0}^{1} d x \frac{1-x}{x^{2}} P_{f}^{q}\left(x, \frac{b}{x}\right)
$$


(a)

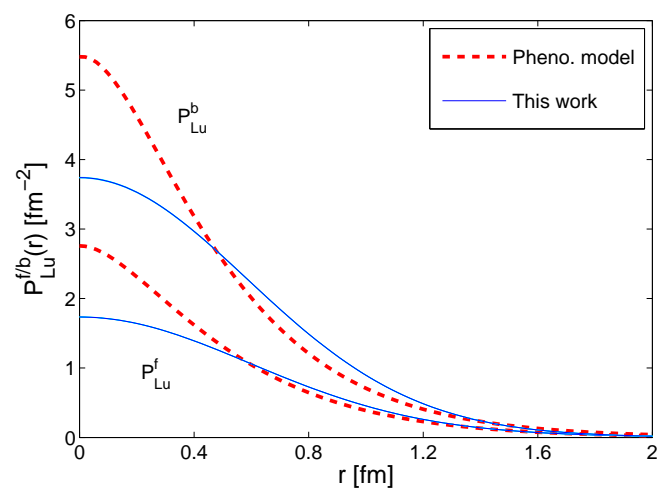

(b)

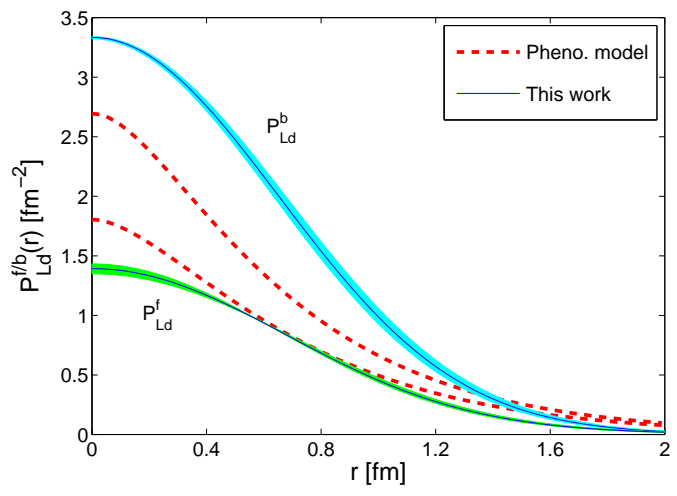

(c)

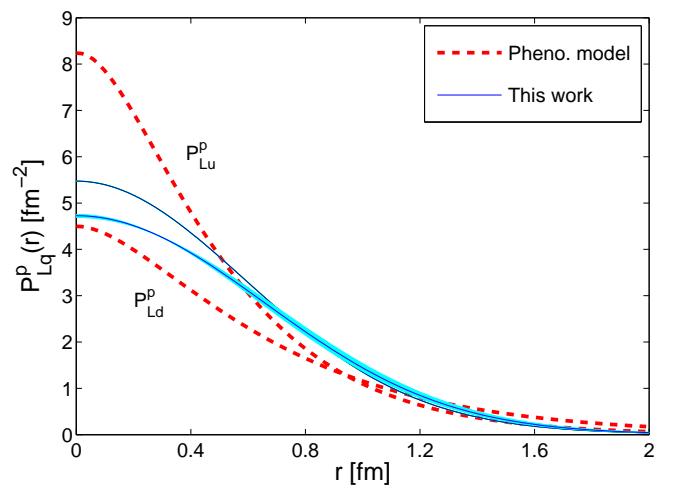

FIG. 5. (colour online) Longitudinal momentum distributions (a) $P_{L}^{f / b}$ for struck $u$ quark, (b) $P_{L}^{f / b}$ for struck $d$ quark $d$ and (c) $P_{L}^{p}$ for proton when struck quarks are $u$ and $d$. The blue solid lines represent the quark-diquark model in AdS/QCD (this work) and the red dashed lines represent the phenomenological model [46]. The error bands shown in the plots are estimated from the errors in the model parameters.

Therefore, the longitudinal momentum distribution in $\mathbf{b}_{\perp}$-space for the nucleons can be expressed as

$$
\rho_{L q}^{N}(b)=\rho_{L q}^{f}(b)+\rho_{L q}^{b}(b) .
$$

The longitudinal distributions in the impact-parameter plane in the light-front quarkdiquark model in AdS/QCD has been investigated in [10] but in the present work we extend the calculations to study the longitudinal momentum distribution in transverse coordinate space. In Fig, we show the longitudinal momentum distributions in transverse coordinate space in the light-front quark-diquark model using the wave functions modeled by soft-wall AdS/QCD. The quark (fermionic) and diquark (bosonic) contributions are shown in Fig 5(a) and 5(b) for different struck $u$ and $d$ quarks respectively. The total distribution for proton (quark + diquark) is shown in Fig 5 (c). The figures show that the diquark contributions 
(a)

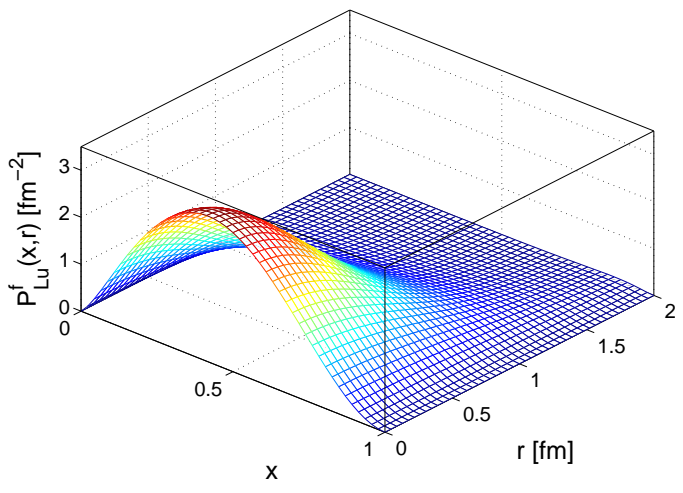

(b)

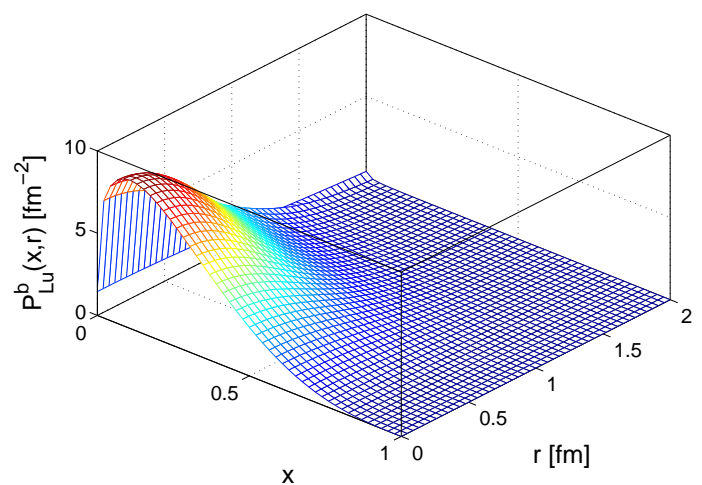

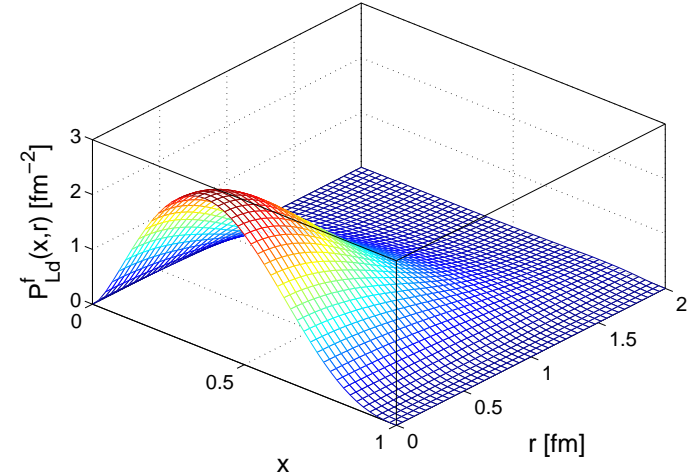

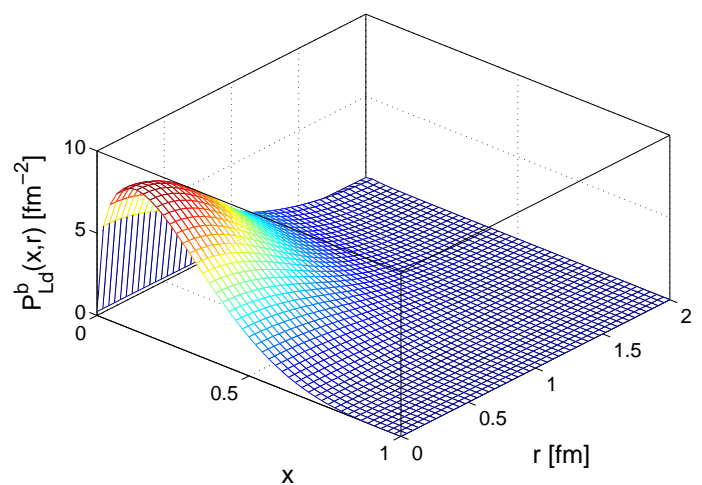

(c)

(d)

FIG. 6. (colour online) Longitudinal momentum distributions $P_{L q}^{f}\left(x, \mathbf{r}_{\perp}\right)$ and $P_{L q}^{b}\left(x, \mathbf{r}_{\perp}\right)$ as a function of $\mathbf{r}_{\perp}$ and $x$. Left panel: when the struck flavor is $u$ and the diquark is $u d$; right panel: when the struck flavor is $d$ and the diquark is $u u$.

are comparatively larger than the quark contributions for both the cases where the struck quarks are $u$ and $d$. Comparing with the phenomenological model, one finds that both the contributions from the quark and diquark to the proton distribution are larger in phenomenological model as compared to that in the quark-diquark model in AdS/QCD when the struck quark is $u$ quark. The total distribution i.e. quark+diquark distribution should be independent of the struck quark. For the case of proton, the total distribution for $u$ struck quark is slightly larger than that for the $d$ struck quarkwhereas the difference between these two distributions is quite large in the phenomenological model considered. So, we can say that the quark-diquark model in $\mathrm{AdS} / \mathrm{QCD}$ is better compared to the phenomenological model considered here. The distribution $P_{L q}$ as a function of $x$ and $r$ for fermionic part as well as the bosonic part considering different struck quark are plotted in Fig.6. We find that the fermionic distributions have the peak near the middle of $x$ whereas for bosonic distributions, the peak shifts to lower $x$. The fall-off of quarks distributions (Fig [6(a) and 


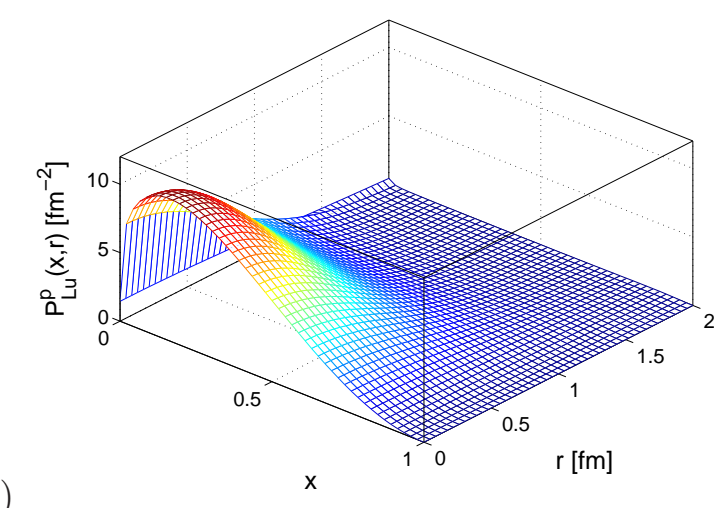

(a)

FIG. 7. (colour online) Longitudinal momentum distributions $P_{L q}\left(x, \mathbf{r}_{\perp}\right)$ for proton as a function of $\mathbf{r}_{\perp}$ and $x$, (a) for struck flavor $u$ and (b) for struck flavor $d$.

Fig $[(\mathrm{b}))$ at large $x$ are slower than the diquark distributions (Fig [6(c) and Fig, $6(\mathrm{~d})$ ). The magnitudes of the diquark distributions are quite large compared to quark distributions. Thus, the peak of the total (quark + diquark) distributions effectively appears at lower $x$ but the fall-off the total distributions are little slower than the diquark distributions as shown in Fig:7. The magnitudes of the total distributions for different struck quarks are almost the same.

\section{SUMMARY AND CONCLUSIONS}

In the present work, we have presented the charge distributions in transverse coordinate space for $u$ and $d$ quark as well as for the nucleons in a light-front quark-diquark model where the wave functions are modeled from the solution of two particle wave function in the soft-wall AdS/QCD correspondence. We have compared the charge distributions with the charge densities in impact-parameter space. A relation between the distributions in transverse coordinate space and the impact-parameter plane has been shown. It has been found that the charge distributions in coordinate plane are more widely spread compared to the impact-parameter distributions. This phenomena can be clearly understood from the relation between these two different distributions which implies that the $\mathbf{r}_{\perp}$ is equivalent to $\frac{\mathbf{b}_{\perp}}{x-1}$ in $P\left(\mathbf{r}_{\perp}\right)$. The distributions $P\left(x, \mathbf{r}_{\perp}\right)$ for the quarks and the nucleons have the peak at lower $x$ values and gradually decrease in magnitude with increasing $x$. The distribution for neutron is mostly negative but it has also a small positive distribution which suggests that the central charge density is negative covered by a positively charged shell. 
We have also studied the longitudinal momentum distributions for proton and the individual distribution for quark (fermionic) and diquark (bosonic) in the transverse coordinate space from the gravitational form factors. It has been observed that the diquark distributions are larger than the quark distributions but when the quark and diquark contributions are added up, they provide more or less same proton distributions for different struck $u$ and $d$ quarks. $P_{L q}\left(x, \mathbf{r}_{\perp}\right)$ for quarks has the peak near the middle value of $x$ but for diquark the peak is at lower $x$ value. The diquark distributions fall faster than the quark distributions at large $x$. Since $P_{L q}\left(x, \mathbf{r}_{\perp}\right)$ for diquarks are large compared to that for quarks, the proton distributions have the peak at small $x$. The distributions however fall slower than the diquark distributions. It is important to mention here that the density in impact parameter space cannot be interpreted as coordinate space density even though both $r$ and $\left|b_{\perp}\right|$ are conjugate to momentum $k$ and momentum transferred $\Delta_{\perp}$ respectively.

\section{Appendix A: Elementary theorems about convolutions and Fourier transforms}

If we have any two functions, $f\left(\mathbf{k}_{\perp}\right)$ and $g\left(\mathbf{k}_{\perp}\right)$ such that

$$
f\left(\mathbf{k}_{\perp}\right)=\int d^{2} \mathbf{r}_{\perp} e^{-i \mathbf{k}_{\perp} \cdot \mathbf{r}_{\perp}} \tilde{f}\left(\mathbf{r}_{\perp}\right), \quad g\left(\mathbf{k}_{\perp}\right)=\int d^{2} \mathbf{r}_{\perp} e^{-i \mathbf{k}_{\perp} \cdot \mathbf{r}_{\perp}} \tilde{g}\left(\mathbf{r}_{\perp}\right),
$$

where $\tilde{f}\left(\mathbf{r}_{\perp}\right)$ and $\tilde{g}\left(\mathbf{r}_{\perp}\right)$ are the Fourier transform of $f\left(\mathbf{k}_{\perp}\right)$ and $g\left(\mathbf{k}_{\perp}\right)$ respectively. Then the form factor which can be written as

$$
G\left(\mathbf{q}_{\perp}\right) \equiv \int \frac{d^{2} \mathbf{k}_{\perp}}{(2 \pi)^{2}} f^{*}\left(\mathbf{k}_{\perp}-a \mathbf{q}_{\perp}\right) g\left(\mathbf{k}_{\perp}\right),
$$

becomes diagonal in Fourier space and we have

$$
\int \frac{d^{2} \mathbf{q}_{\perp}}{(2 \pi)^{2}} e^{i \mathbf{q}_{\perp} \cdot \mathbf{b}_{\perp}} G\left(\mathbf{q}_{\perp}\right)=\frac{1}{|a|^{2}} \tilde{f}^{*}\left(\frac{\mathbf{b}_{\perp}}{a}\right) \tilde{g}\left(\frac{\mathbf{b}_{\perp}}{a}\right) .
$$

[1] V. Yu. Petrov, P. V. Pobylitsa, M. V. Polyakov, I. Börnig, K. Goeke, and C. Weiss, Phys. Rev. D 57, 4325 (1998).

[2] M. Penttinen, M. V. Polyakov, and K. Goeke, Phys. Rev. D 62, 014024 (2000).

[3] A. Chodos, R. L. Jaffe, K. Johnson, C. B. Thorn, and V. F. Weisskopf, Phys. Rev. D 9, 3471 (1974). 
[4] X. Ji, W. Melnitchouk, and X. Song, Phys. Rev. D 56, 5511 (2000).

[5] T. Gutsche, V. E. Lyubovitskij, I. Schmidt, and A. Vega, Phys. Rev. D 91, 054028 (2015).

[6] T. Gutsche, V. E. Lyubovitskij, I. Schmidt, and A. Vega, Phys. Rev. D 89, 054033 (2014).

[7] D. Chakrabarti and C. Mondal, Phys. Rev. D 92, 074012 (2015).

[8] C. Mondal and D. Chakrabarti, Few Body Syst. 57, no. 8, 723 (2016).

[9] C. Mondal and D. Chakrabarti, Eur. Phys. J. C 75, 261 (2015).

[10] D. Chakrabarti, C. Mondal, and A. Mukherjee, Phys. Rev. D 91, 114026. (2015).

[11] T. Maji, C. Mondal, D. Chakrabarti and O. V. Teryaev, JHEP 1601, 165 (2016).

[12] D. Chakrabarti and C. Mondal, Eur. Phys. J. A 52, 285 (2016).

[13] Z. Abidin and C. E. Carlson, Phys. Rev. D 79, 115003 (2009).

[14] H. Forkel, M. Beyer and T. Frederico, Int. J. Mod. Phys. E 16, 2794 (2007), W. de Paula, T. Frederico, H. Forkel, and M. Beyer, Phys. Rev. D 79, 75019 (2009).

[15] A. Vega, I. Schimdt, T. Gutsche, and V. E. Lyubovitskij, Phys. Rev. D 83, 036001 (2011).

[16] A. Vega, I. Schimdt, T. Gutsche, and V. E. Lyubovitskij, Phys. Rev. D 83, 036001 (2011); Phys. Rev. D 85, 096004 (2012); T. Gutsche, V. E. Lyubovitskij, I. Schmidt, and A. Vega, Phys. Rev. D 86, 036007 (2012).

[17] Z. Abidin and C. E. Carlson, Phys. Rev. D 77, 095007 (2008).

[18] S. J. Brodsky and G. F. de Téramond, Phys. Rev. D 77, 056007 (2008); Phys. Rev. D 78, $025032(2008)$.

[19] S. J. Brodsky and G. F. de Téramond, Phys. Rev. D 83, 036011(2011), Phys. Rev D 85, 096004 (2012).

[20] S. J. Brodsky and G. F. de Téramond, arXiv:1203.4025 [hep-ph].

[21] H. R. Grigoryan and A. V. Radyushkin, Phys. Rev. D 76, 095007 (2007).

[22] A. Vega, I Schmidt, T. Branz, T. Gutsche, and V. E. Lyubovitskij, Phys. Rev. D 80, 055014 (2009); T. Branz, T. Gutsche, V. E. Lyubovitskij, I. Schmidt, and A. Vega, Phys. Rev. D 82, 074022 (2010); T. Gutsche, V. E. Lyubovitskij, I. Schmidt, and A. Vega, Phys. Rev. D 85, 076003 (2012); Phys. Rev. D 87, 056001 (2013); Phys. Rev. D 90, 096007 (2014).

[23] D. Chakrabarti and C. Mondal, Phys. Rev. D 88, 073006 (2013).

[24] D. Chakrabarti and C. Mondal, Eur. Phys. J. C 73, 2671 (2013).

[25] D. Chakrabarti and C. Mondal, Eur. Phys. J. C 74, 2962 (2014).

[26] C. Mondal, Eur. Phys. J. C 76, no. 2, 74 (2016). 
[27] K. Hashimoto, T. Sakai, and S. Sugimoto, Prog. Theor. Phys. 120, 1093 (2008).

[28] Z. Abidin and C. E. Carlson, Phys. Rev. D 78, 071502 (2008).

[29] S. J. Brodsky, G. F. de Teramond, H. G. Dosch, and J. Erlich, Phys. Rept. 584, 1 (2015).

[30] N. Kumar and H. Dahiya, Phys. Rev. D 90, 094030 (2014).

[31] T. Gutsche, V. E. Lyubovitskij, I. Schmidt, and A. Vega, Phys. Rev. D 87, 016017 (2013).

[32] J. M. Maldacena, Adv. Theor. Math. Phys. 2, 231 (1998); Int. J. Theor. Phys. 38, 1113 (1999).

[33] S. J. Brodsky and G. F. de Teramond, Phys. Lett. B 582, 211 (2004).

[34] S. J. Brodsky and G. F. de Teramond, Phys. Rev. Lett. 96, 201601 (2006), Phys. Rev. D 77, 056007 (2006).

[35] S. J. Brodsky and S. D. Drell, Phys. Rev. D 22, 2236 (1980).

[36] S. J. Brodsky and D. S. Hwang, Nucl. Phys. B 543, 239 (1999); S. J. Brodsky, D. S. Hwang, B. -Q. Ma, and I. Schmidt, Nucl. Phys. B 593, 311 (2001); S. J. Brodsky, M. Diehl, and D. S. Hwang, Nucl. Phys. B 596, 99 (2001).

[37] G. A. Miller, Phys. Rev. Lett. 99, 112001 (2007).

[38] C. E. Carlson and M. Vanderhaeghen, Phys. Rev. Lett. 100, 032004 (2008).

[39] C. Granados, C. Weiss, JHEP 1401, 092 (2014).

[40] O. V. Selyugin and O.V. Teryaev, Phys. Rev. D79, 033003 (2009).

[41] M. Burkardt, Phys. Rev D. 62, 071503 (2000), Int. J. Mod. Phys. A 18, 173 (2003).

[42] G. A. Miller, Phys. Rev. C 80, 045201 (2009).

[43] G. A. Miler, Annu. Rev. Nucl. Part. Sci. 60, 1 (2010).

[44] S. Venkat, J. Arrington, G. A. Miller, and X. Zhan, Phys. Rev. C 83, 015203 (2011).

[45] M. Burkardt, Phys. Rev. D 62, 071503 (2000).

[46] D. S. Hwang, D. S. Kim and J. Kim, Phys. Lett. B 669, 345 (2008).

[47] N. Kumar and H. Dahiya, Mod. Phys. Lett. A 29, 1450118 (2014).

[48] T. Liu and B. Q. Ma, Int. Jou. Mod. Phys: Conf. Series 40, 1660054 (2016).

[49] D. Muller et al., Fortschr. Phys. 42, 101 (1994); X. Ji, Phys. Rev. Lett. 78, 610 (1997); A.V. Radyushkin, Phys. Rev. D 56, 5524 (1997).

[50] X. Ji, W. Melnithouk, and X. Song, Phys. Rev. D 56, 5511 (1997).

[51] S. J. Brodsky and G. F. de Téramond, Phys. Rev. D 78, 025032 (2008).

[52] Z. Abidin and C. E. Carlson, Phys. Rev. D 77, 115021 (2008). 